VERSITA

Biometrical Letters

DOI:10.2478/bile-2013-0007

Vol. 49 (2012), No. 2, 95-102

\title{
On the relevance of the $3 \mathrm{D}$ retinal vascular network from OCT data
}

\author{
P. Serranho ${ }^{1,2}$, P. Guimarães ${ }^{2}$, P. Rodrigues ${ }^{3}$, R. Bernardes ${ }^{2,3}$ \\ ${ }^{1}$ Department of Science and Technology, Portuguese Open University, Portugal, \\ e-mail: pserranho@uab.pt \\ ${ }^{2}$ Institute of Biomedical Research in Light and Image, Faculty of Medicine, University \\ of Coimbra, Coimbra, Portugal \\ ${ }^{3}$ CNTM, Association for Innovation and Biomedical Research on Light and Image, \\ Coimbra, Portugal
}

\section{SUMmARY}

\begin{abstract}
Abnormal patterns of the retinal vascular system have been associated with several heart and cerebral diseases. However, these correlations were obtained using 2D imaging of the eye fundus, disregarding information on the third component (depth) of the vascular system. In this paper, we show that the depth component is relevant and should not be disregarded in this analysis. In this way, the relevance of analysis of the retinal vascular network as a biometrical marker for the general health status of an individual can be increased.
\end{abstract}

Key words: biometrical marker, 3d retinal vascular network, optical coherence tomography data

\section{Introduction}

The retinal vascular system is usually imaged using color fundus photography (CFP) or fluorescein angiography (FA), both providing only a $2 \mathrm{D}$ projection of the retinal vascular system. Moreover, FA is an invasive technique, as it relies in the administration of a fluorescent dye, which causes provoques adverse reactions in about $5 \%$ of cases (Laatikainen, 2006). From the retinal vascular system as imaged by these modalities, one can retrieve several metrics, such as tortuosity, length, diameter and angle of bifurcation, among others (Bhuiyan et al., 2007). It is clear that if these metrics are obtained from 2D projections, disregarding the depth component, there is loss of information. Nonetheless, correlations between these metrics and heart (Witt et al., 2006) and cerebral diseases (Kwa et al., 2002, Patton et $a l ., 2005)$ have been shown, as well as in the cases of diabetes and hypertension (Wang et al., 2005). 
Optical coherence tomography (OCT) is an in vivo imaging modality of the human eye fundus. Making use of low coherent light interferometry, it provides 3D volumetric data for light backscattering of the human eye fundus, making it possible to image the human retina non-invasively. OCT has been mainly used in a clinical sense to diagnose macular edema, or in other words to establish a thickness map of the retina. However, in a series of recent projects by our research group (Guimarães et al., 2012, Guimarães et al., 2012b, Rodrigues et al., 2012), significant progress has been made in recovering the 3D positioning of the retinal vascular network. This possibility makes use of the fact that blood absorbs light at the wavelengths used in OCT (Chen et al., 2003, Fabritius et al., 2009), therefore provoking a shadow in the depth-wise direction below a blood vessel in the 3D OCT data.

Recent work shows that about $70 \%$ of the retinal vascular system recovered from FA can be recovered from OCT data (Rodrigues et al., 2012). Moreover, ongoing research shows that it is possible to recover the $3 \mathrm{D}$ position of the retinal vascular system from OCT (Guimarães et al., 2012, Guimarães et al., 2012b).

Kiss et al. (2005) have already mentioned the importance of obtaining 3D information on the retinal vascular system, but disclose no further information. It is relevant to know whether the depth component of the position of the vascular system can change significantly the metrics used and therefore the correlations with disease status. If this is the case, the use of 3D metrics might strengthen correlations between the retinal vascular system and the corresponding disease status of the subject.

In this paper we test the significance of the correlations between widely used tortuosity metrics in $2 \mathrm{D}$ and $3 \mathrm{D}$, for the same subject. We will consider correlations between metrics in 2D and 3D and between metrics in $2 \mathrm{D}$ and in the depth component alone. The latter indicates whether tortuosity in $2 \mathrm{D}$ and in the depth component are dependent. If so, there should be no relevance in the $3 \mathrm{D}$ segmentation for correlation purposes. However, if the tortuosity on the $2 \mathrm{D}$ projection and the depth component alone are not correlated, the new information on the tortuosity in depth might provide new correlations with disease status.

The paper is organized as follows. In section 2, a brief overview of the $3 \mathrm{D}$ segmentation method for the retinal vascular network is given. In section 3 we present the tortuosity metrics used and formulate the statistical problem that we wish to answer, that is, wheather the $2 \mathrm{D}$ and $3 \mathrm{D}$ metrics 


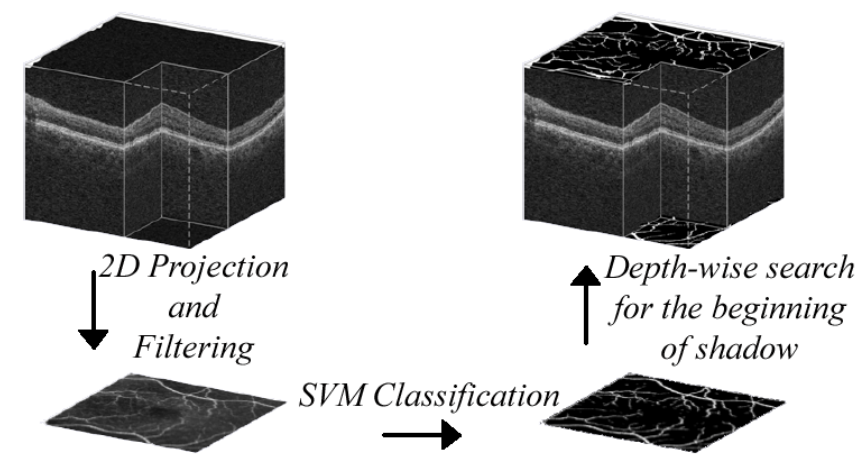

Figure 1. Scheme of the $3 \mathrm{D}$ retinal vascular system segmentation process from OCT data

are significantly correlated. In section 4 we present the main results, and in section 5 we summarize the main conclusions and make some references to future work.

\section{Vascular Network Segmentation}

We will provide only a brief overview of segmentation in 2D and 3D. Details can be found in Guimarães et al. (2012).

We first consider the appropriate projection of 3D volumetric OCT data to obtain $2 \mathrm{D}$ projections of the vascular system. To achieve this, we make use of the depth-wise shadow caused by the vessel in the OCT signal, in order to get several images with good contrast between vessel and nonvessel pixels. These images are then processed with several filters, based on intensity, gradient and Hessian, among others. Local phase features are also computed.

This set of images is used to obtain a binary segmented image by means of Support Vector Machine (SVM) classification as detailed in Guimarães et al. (2012).

Having the 2D projection of the vessels, the depth component is obtained by depth-wise search of the start of the vessel shadow in the OCT data. This procedure is diagrammed in Figure 1.

\section{Tortuosity metrics and correlation}

From the vessel segmentation, one retrieves a binary image where true and false pixels correspond respectively to the presence or absence of a vessel. 
The vessel centerlines were retrieved. Tortuosity metrics were computed for all vessel segments defined as the path between two endpoints, an endpoint and a branchpoint, or two branchpoints. Note that an endpoint is a vessel point with at most one vessel point in its $3 \times 3$ neighborhood, while a branchpoint has at least three (these may include bifurcation or crossovers).

The fundus image is convoluted with a bank of log-Gabor filters, each having its unique direction-scale pair. Then each vessel segment was categorized into thin, medium or large, depending on the average scale with maximum filter response along the segment.

Three different metrics were used to assess vessel tortuosity: the distance metric (DM), the sum of angles metric (SOAM), and the inflection count metric (ICM). To our knowledge these are the main vessel tortuosity metrics used in the literature (see for instance Bullit et al., 2003, Parikh et al., 2004, Truco et al., 2010, among others).

The DM is the most common approach when evaluating vessel tortuosity. It is the ratio between the total length of the vessel and the linear distance between its ends. However, this metric has shown that it cannot completely describe vessel tortuosity. For instance, it cannot distinguish a curve from a vessel that has abrupt direction changes.

The SOAM can be described as the integrated total curvature normalized by the path length. The ICM is the total number of inflection points multiplied by DM. These metrics were computed as described in Bullit et al. (2003). Instead of using just the adjacent points $P_{k-1}$ and $P_{k+1}$ at each point $P_{k}$, the four points $P_{k-s}, P_{k}, P_{k+s}, P_{k+2 s}$ were considered, where $s$ is the spacing between the points.

Since a pixel representation is used to describe the blood vessels (a discrete representation), undesirable noise can be introduced into metrics such as SOAM and ICM. For instance, a diagonal vessel with respect to the sampling direction of the OCT can be represented as ragged line. Thus the vessel representation must be smoothed prior to the metric computation. Instead of computing these metrics on the vessel path itself, for each point $\mathrm{Pk}$, a polynomial (maximum order 3 ) least square fit with the path $P_{k-s}, P_{k}, P_{k+s}, P_{k+2 s}$ was computed independently for coordinates x, $\mathrm{y}$ and $\mathrm{z}$.

In the first approach, the comparison was performed between the $2 \mathrm{D}$ and 3D metrics. Although, to our surprise, this turned out to be not completely the case, it was expected that no major differences would be found. This expectation can be explained due to the nature of the retina 


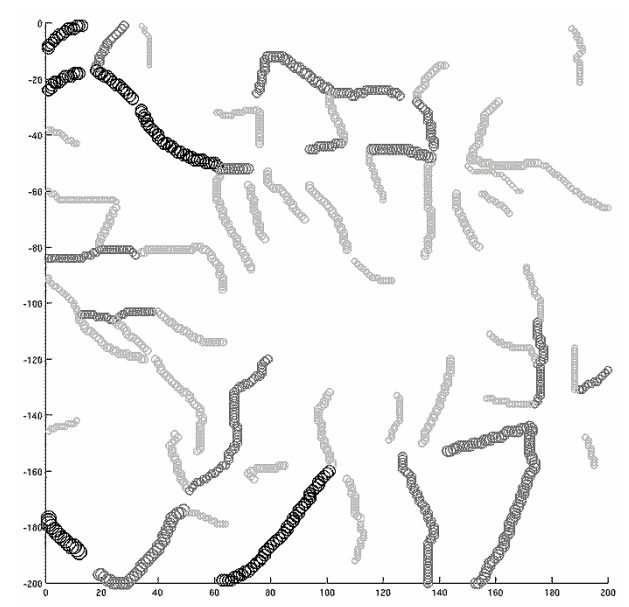

Figure 2. The recovered network by vessel diameter: thin, medium and large vessels. Small branches were eliminated from the segmentation

itself. The retina presents an macular thickness that ranges between about 141 micrometers in the foveal area and about 267 micrometers in the outer macular areas (Pires et al., 2002), while the scans were retrieved from an area of 6000x6000 micrometers (x and y directions). This can mean that depth changes in vessel position are lost due to their small scale. Hence in the second approach, 2D projection tortuosity was compared to the tortuosity in depth.

To achieve our purpose, we began by dividing the recovered vessels for each subject according to their diameter. Therefore we considered three classes of vessels: thin, medium and large (see Figure 2), depending on the diameter of the vessel in pixels and the OCT protocol used in the acquisition of the image.

In each of these classes we establish the correlation $R$ between the values of the three metrics (DM, ICM, SOAM) in $2 \mathrm{D}$ and $3 \mathrm{D}$, in order to test whether the correspondence is linear. From there, one can establish whether the linear regression is significant, that is, considering the linear regression line

$$
\hat{y}=\beta_{0} x+\beta_{1}
$$

we considered the hypothesis test

$$
\left\{\begin{array}{l}
\mathrm{H}_{0}: \beta_{0}=0 \\
\mathrm{H}_{1}: \beta_{0} \neq 0
\end{array}\right.
$$


It can be shown that this is equivalent to a t-Student test given by

$$
\frac{R}{\sqrt{\frac{1-R^{2}}{n-2}}} \sim t_{1-\alpha / 2, n-2}
$$

where $n$ is the dimension of the sample. From there, if the $p$-value was lower than the level of significance $\alpha$, there was a significant linear relation between the values in $2 \mathrm{D}$ and $3 \mathrm{D}$, while in the other case there was not statistical evidence of a linear relation. The latter means that there is a possible change in the correlations between $2 \mathrm{D}$ and $3 \mathrm{D}$ metrics and disease status.

\section{Results}

We considered 15 OCT volumes from healthy and early stage diabetic patients. We also considered a significance level of $\alpha=0.05$.

We began by comparing the results between the tortuosity metrics in $2 \mathrm{D}$ and $3 \mathrm{D}$. In Table 1 we present the obtained correlations $R$ and $p$-values for the hypothesis test (1). It is clear that for DM in small vessels the null hypothesis is accepted, that is, there is no statistical evidence that the relation is linear. In fact, this shows that the most widely used tortuosity metric (DM) is significantly different in $2 \mathrm{D}$ and $3 \mathrm{D}$ as concerns the retinal vascular system. The difference is greater in smaller vessels, since they tend to be more tortuous in depth.

Table 1. Table of correlations between 2D and 3D tortuosity metrics and the respective $p$-values for the hypothesis test (1)

\begin{tabular}{ccccccc}
\hline Diameter & \multicolumn{3}{c}{ Correlation $R$} & \multicolumn{3}{c}{$p$-value } \\
& DM & SOAM & ICM & DM & SOAM & ICM \\
\hline thin & -0.459 & 0.957 & 0.612 & $\mathbf{0 . 0 8 5}$ & $<0.001$ & 0.015 \\
medium & 0.238 & 0.991 & 0.960 & $\mathbf{0 . 3 9 4}$ & $<0.001$ & $<0.001$ \\
large & 0.535 & 0.949 & 0.993 & 0.040 & $<0.001$ & $<0.001$ \\
\hline
\end{tabular}

Taking into consideration the relatively small retinal thickness, we compared the still significant tortuosity metrics (SOAM, ICM) using 2D and only the depth-wise data. The results are shown in Table 2 . In this case it is clear that the SOAM also becomes nonlinear, therefore opening up the possibility of using the depth-wise tortuosity alone to correlate with disease status. 
On the relevance of the $3 \mathrm{D}$ retinal vascular network from OCT data

Table 2. Table of correlations between $2 \mathrm{D}$ and depth-wise (only) tortuosity metrics and the respective $p$-values for the hypothesis test (1)

\begin{tabular}{ccccc}
\hline Diameter & \multicolumn{2}{c}{ Correlation $R$} & \multicolumn{2}{c}{$p$-value } \\
& SOAM & ICM & SOAM & ICM \\
\hline thin & 0.228 & 0.596 & $\mathbf{0 . 4 1 3}$ & 0.019 \\
medium & 0.485 & 0.818 & $\mathbf{0 . 0 6 7}$ & $<0.001$ \\
large & 0.829 & 0.967 & $<0.001$ & $<0.001$ \\
\hline
\end{tabular}

\section{Conclusions and future perspectives}

We intended to show that tortuosity metrics in 2D (that is, disregarding the depth component) do not relate linearly with tortuosity metrics considering the depth-wise component, as concerns the retinal vascular system.

In this preliminary study, we show that the most widely used tortuosity metric (DM) for measuring vessel tortuosity is one where the linear relation between $2 \mathrm{D}$ and $3 \mathrm{D}$ metrics is not significant. This shows that the use of 3D tortuosity metrics to correlate with disease status might have a significant impact on the correlation values, when compared with those obtained using $2 \mathrm{D}$ metrics.

The next step is to investigate whether this change comes in the beneficial to diagnosis, that is, whether the correlations with disease status become stronger using $3 \mathrm{D}$ metrics rather than $2 \mathrm{D}$ metrics. This will be the subject of future work.

\section{Acknowledgments}

This work was partially supported by the Portuguese Foundation for Science and Technology (Fundação para a Ciência e a Tecnologia) under the research project PTDC/SAU-ENB/111139/2009 and the program COMPETE (FCOMP-01-0124-FEDER-015712).

\section{REFERENCES}

Bhuiyan A., Nath B., Chua J., Ramamohanarao K. (2007): Automatic Detection of Vascular Bifurcations and Crossovers from Color Retinal Fundus Images, Third International IEEE Conference on Signal-Image Technologies and Internet-Based System: 711-718.

Bullitt E, Gerig G, Pizer S, Aylward S.R. (2003): Measuring Tortuosity of the Intracerebral Vasculature from MRA Images. IEEE-TMI 22(9): 1163-1171.

Chen Z., Kai Y., Ge H., Xu Z. (2003): The study on the light absorption and transmission laws of the blood components, Third Intern. Conf. on Photonics and Imaging in Biology and Medicine. Edited by Luo, Qingming; Tuchin, Valery V.; Gu, Min;Wang, Lihong V. Proceedings of the SPIE 5254: 257-261. 
Fabritius T., Makita S., Hong Y., Myllyla R., Yasuno Y. (2009): Automated retinal shadow compensation of optical coherence tomography images, J. Biomed. Opt. 14(1), 010503, doi: 10.1117/1.3076204

Guimarães P., Rodrigues P., Serranho P., Berbardes R. (2012): 3D Retinal Vascular Network from Optical Coherence Tomography Data. Image Analysis and Recognition, Lecture Notes in Computer Science. Image Analysis and Recognition, Springer Berlin/Heidelberg, 7325: 339-346.

Guimarães P., Rodrigues P., Serranho P., Bernardes R. (2012): 3D Retinal Vascular Network from OCT data. ARVO Annual Meeting, May 6-10, 2012, Fort Lauderdale, USA.

Kiss C.G., Leitgeb R., Michels S., Hermann B., Ahlers C., Povazay B., Sacu S., Sattmann H., Schmidt-Erfurth U., Drexler W. (2005): Retinal Vasculature Imaging Using Three Dimensional Ultra-High Resolution Optical Coherence Tomography.Invest. Ophthalmol. Vis. Sci. 46: E-Abstract 3467

Kwa, V.I.H., van der Sande J.J., Stam J., Tijmes N., Vrooland J.L. (2002): Retinal arterial changes correlate with cerebral small-vessel disease. Neurology 59: $1536-1540$.

Laatikainen L. (2006): Adverse effects of fluorescein angiography Acta Ophthalmologica Scandinavica 84(6): 720-721.

Parikh A.H., Smith J.K., Ewend M.G., Bullitt E. (2004): Correlation of MR Perfusion Imaging and Vessel Tortuosity Parameters in Assessment of Intracranial Neoplasms. Technology in Cancer Research \& Treatment 3(6): 585-590.

Patton N., Aslam T, Macgillivray T, Pattie A, Deary IJ, Dhillon B. (2005): Retinal vascular image analysis as a potential screening tool for cerebrovascular disease: a rationale based on homology between cerebral and retinal microvasculatures. J Anat. 206(4): 319-348.

Pires I., Bernardes R.C., Lobo C.L., Soares M.A., Cunha-Vaz J.G. (2002): Retinal thickness in eyes with mild nonproliferative retinopathy in patients with type 2 diabetes mellitus. Comparison of measurements obtained by retinal thickness analysis and optical coherence tomography. Arch. Ophthalmol. 120: 1301-1306.

Rodrigues P., Guimarães P., Bernardes R., Serranho P. (2012): Vascular Network of the human macula from high-definition OCT. ARVO Annual Meeting, May 6-10, 2012, Fort Lauderdale, USA.

Truco E., Azegrouz H., Dhillon B. (2010): Modeling the Tortuosity of Retinal Vessels: Does Caliber Play a Role?, IEEE Trans. Biomed Eng. 57(9): 2239-2247.

Wang X., Cao H., Zhang J. (2005): Analysis of Retinal Images Associated with Hypertension and Diabetes. Engineering in Medicine and Biology Society, 2005. IEEE-EMBS 2005. 27th Annual International Conference of the: 6407-6410.

Witt N., Wong T.Y., Hughes A.D., Chaturvedi N., Klein B.E., Evans R., McNamara M., McG Thom S.A., Klein R. (2006): Abnormalities of Retinal Microvascular Structure and Risk of Mortality From Ischemic Heart Disease and Stroke. Hypertension 47: 975-981. 